\title{
Clinical Study on Different Types of Insomnia Based on Conducting Yin from Yang, Conducting Yang from Yin Method with Spleen and Stomach as Center
}

\author{
Bian Wei ${ }^{1}$, Wang Huanqun ${ }^{2,}$, Zou Weiwu ${ }^{2}$, Gan Ting ${ }^{2}$ \\ ${ }^{1}$ Department of Rehabilitation, Chongqing Yongchuan Hospital of Traditional Chinese Medicine, Chongqing, China \\ ${ }^{2}$ Department of Neurology, Chongqing Yongchuan Hospital of Traditional Chinese Medicine, Chongqing, China
}

Email address:

2380139344@qq.com (Bian Wei), 554132994@qq.com (Wang Huanqun)

${ }^{*}$ Corresponding author

To cite this article:

Bian Wei, Wang Huanqun, Zou Weiwu, Gan Ting. Clinical Study on Different Types of Insomnia Based on Conducting Yin from Yang, Conducting Yang from Yin Method with Spleen and Stomach as Center. International Journal of Chinese Medicine.

Vol. 4, No. 3, 2020, pp. 61-66. doi: 10.11648/j.ijcm.20200403.13

Received: August 17, 2020; Accepted: September 8, 2020; Published: September 19, 2020

\begin{abstract}
Objective] On the basis of observing Jinding Decoction (Glycyrrhiza 6g Poria 9g Pinellia 3g Guizhi 9g Peony $9 \mathrm{~g}$ Long Bone $6 \mathrm{~g}$ Oyster 9g), clinical research on different types of insomnia based on the pulse condition. [Method] Using the randomized parallel control method, 150 patients with insomnia who were treated in the Treatment Center and Brain Disease Department of our hospital from November 2019 to June 2020 were divided into treatment group, TCM control group, Western medicine control group, and TCM control group Adopting traditional Chinese medicine to collect through observation, hearing, and inquiring. According to the symptoms, tongue condition and pulse condition, the syndromes were differentiated one by one, followed by TCM classification and treatment. The western medicine control group was treated with alprazolam tablets (Jiangsu Enhua Pharmaceutical Co., Ltd., Zhunzi H32020215). Medicine treatment. 0.4 mg daily, orally 30 minutes before bedtime. Continuous treatment for 10 days is a course of treatment, each course is separated by 1 day, a total of 3 courses of treatment, observation of long-term efficacy observation before and after treatment were performed 1 day before the beginning of the treatment, the second day after the end of the treatment, and one month after stopping the treatment. Patients in the group were evaluated for clinical efficacy, PSQI score, and (SLC-90) score. [Results] Jinding Decoction had statistical significance in the treatment of insomnia of various syndrome types $(\mathrm{P}<0.05)$. [Conclusion] Spleen and stomach harmony is the basis for the orderly rise and fall of yin and yang qi, the hidden primordial spirit, and the foundation of sleep soundness. Jinding Decoction is based on the basic theory of traditional Chinese medicine.
\end{abstract}

Keywords: Clinical Study, Different Types of Insomnia, Spleen and Stomach as Center

\section{Introduction}

We believe that yin and yang imbalance is the total pathogenesis of insomnia. According to the traditional Chinese medicine theory " harmonizing stomach to tranquilize the mind and the theory that disharmony of stomach leads to insomnia ", and the western medicine brain-intestinal theory: the gastrointestinal nervous system is the human "second brain", the traditional Chinese medicine theory of the spleen and stomach center and Western medicine is closely related to the gastrointestinal nerves. Using the randomized parallel control method, 150 patients with insomnia who were treated in the Treatment Center and Brain Disease Department of our hospital from November 2019 to June 2020 were divided into treatment group, TCM control group, Western medicine control group, and TCM control group Adopting traditional Chinese medicine to collect through observation, hearing, and inquiring. According to the symptoms, tongue condition and pulse condition, the syndromes were differentiated one by one, followed by TCM classification and treatment. The western medicine control group was treated with alprazolam tablets (Jiangsu Enhua Pharmaceutical Co., Ltd., Zhunzi H32020215). Medicine treatment. $0.4 \mathrm{mg}$ daily, orally 30 minutes before bedtime. Continuous treatment for 10 days is a course of 
treatment, each course is separated by 1 day, a total of 3 courses of treatment, observation of long-term efficacy observation before and after treatment were performed 1 day before the beginning of the treatment, the second day after the end of the treatment, and one month after stopping the treatment. Patients in the group were evaluated for clinical efficacy, PSQI score, and (SLC-90) score. The report is as follows.

\section{Materials and Methods}

\subsection{General Information}

Selected from October 2019 to June 2020, 150 patients with insomnia in the Prevention Treatment Center and Department of Brain Diseases, Chongqing Yongchuan Hospital of Traditional Chinese Medicine, were randomly divided into TCM control group, TCM treatment group, and Western medicine control group. The three groups were in gender, age, and There was no statistically significant difference in the course of disease $(\mathrm{P}>0.05)$.

\subsection{Diagnostic Criteria}

\subsubsection{Western Medicine Diagnosis}

Refer to the "Chinese Mental Disorder Classification and Diagnosis Criteria 3rd Edition (CCM D-3)" Formulated in 2001 by the Psychiatric Branch of the Chinese Medical Association [1].

1) Almost insomnia is the only symptom, including difficulty falling asleep, lack of sleep, multiple dreams, early awakening, or difficulty sleeping again after waking up, discomfort, fatigue, or daytime sleepiness after waking up;

2) Insomnia and extreme attention The dominant concept of insomnia results;

3) Dissatisfaction with the quantity and quality of sleep in severe cases causes obvious distress or impaired social function;

4) At least 3 times a week and at least 1 month;

5) Exclude physical diseases or Secondary insomnia caused by symptoms of mental disorders.

\subsection{2. "Diagnosis of Traditional Chinese Medicine"}

According to the 2012 Edition of the State Administration of Traditional Chinese Medicine "Diagnosis and Efficacy Standards of Chinese Medicine Diseases" [2].

(1) Deficiency of the heart and spleen: In addition to the symptoms of poor sleep, there are also heart palpitations, forgetfulness, dizziness, dreaminess, easy awakening, tired limbs, tasteless diet, poor complexion, pale tongue, thin coating, weak pulse;

(2) fire excess from Yin deficiency: In addition to upset and insomnia, throbbing and restlessness, dizziness, tinnitus, forgetfulness, backache, hot hands, feet and heart, red tongue, pulse count;

(3) timidity due to deficiency of heart qi: In addition to dreams, easy awakening, timid and Heart palpitations, frightened by things, shortness of breath, fatigue, clear and long urine, pale tongue, thin pulse;

(4) phlegm-fire disturbing heart: In addition to insomnia, heavy head, phlegm, chest tightness, Belching, Pantothenic acid, dizziness, greasy and yellow fur, slippery pulse;

(5) Liver-Qi Stagnation: In addition to insomnia, there are impatient temperament, irritability, singing, chest and hypochondriac pain, unfixed pain, abdominal distension, poor appetite, abnormal stool, thin and greasy coating, and wiry pulse.

\subsection{Inclusion Criteria}

\subsubsection{Inclusion Criteria for the Control Group and} Observation Group of Traditional Chinese Medicine

1) Patients Who Meet the Appealed Western Medicine Diagnostic Criteria for Insomnia, with a Course of More Than 3 Months

2) Patients Who Meet the Diagnostic Criteria of Chinese Medicine for Insomnia, with a Course of More Than 3 Months

3) The Mental State Is Normal, and the MMSE is Within the Normal Range

4) Willing to Accept the Questionnaire Survey and Sign the Consent Form

All the above conditions are met to be included in this research.

\subsubsection{Inclusion Criteria of Western Medicine Control Group}

i). Adults who are generally healthy and without sleep disorders;

ii). Those who have no serious mental disorders or physical diseases;

iii). Not taking or using drugs that may significantly affect sleep, etc.;

iv). Willing to accept the questionnaire survey and sign the consent form.

All of the above conditions can be included in this study.

\subsection{Exclusion Criteria}

(1) Patients with Organic Damage Diseases Such as Severe Heart Disease, Lung Disease, Digestive Tract Disease, Thyroid Dysfunction, and Tumor

(2) Patients Whose Sleep May be Significantly Affected by Taking Other Drugs in the Near Future

(3) Non-cooperative Persons

Satisfying any of the above will exclude this research.

\subsection{Method}

\subsubsection{Treatment}

A total of 150 patients with insomnia who were treated in the Treatment Center and Brain Disease Department of our hospital from November 2019 to June 2020 were divided into 3 groups, with 50 cases in each. In the control group of Chinese medicine, 50 cases of TCM were collected through observation and inquiries to analyze the living habits of patients with insomnia, 
the physique of TCM and other influencing factors. The syndromes were differentiated one by one according to symptoms, tongue and pulse conditions. Insomnia was divided into 5 TCM syndrome types (according to the 2012 edition of the National Chinese Medicine). Medical Administration "Standards for Diagnosis and Efficacy of TCM Symptoms"): Deficiency of both heart and spleen, Yin deficiency and fire, heart deficiency and timidity, phlegm and fire disturbing the heart, liver stagnation and qi stagnation, and are classified by Chinese medicine and treated according to syndrome differentiation. The corresponding decoctions are as follows: (1) Subjects with deficiency of both heart and spleen receive Guipi Decoction; (2) Subjects with Yin deficiency and hyperactivity receive Huanglian Ejiao Decoction; (3) Subjects with deficiency of heart and spleen receive Anshen Dingzhi Pill. (4) Subjects with phlegm and fire disturbing the heart receive Huanglian Wendan Decoction; (5) Subjects with liver stagnation and qi stagnation receive Xiaoyao pills; Chinese medicine is provided by Yongchuan District Chinese Pharmacy, unified by Yongchuan District Chinese Medicine Hospital Decoction Boiled. The western medicine control group was treated with alprazolam tablets (Jiangsu Enhua Pharmaceutical Co., Ltd., Chinese Medicine Zhunzi H32020215) as a single agent. $0.4 \mathrm{mg}$ daily, orally 30 minutes before bedtime. Based on the Jinding Decoction (Glycyrrhiza $6 \mathrm{~g}$ Poria 9g Pinellia $3 g$ Cinnamon sticks 9g Peony 9g keel 6g Oyster 9g), 50 cases in the treatment group were based on the pulse condition. Normal; Adding Ophiopogon, Astragalus, Radix Atractylodes, Fritillaria, etc. to you Cunbujiang restores the qi of the lungs to normal; Adding Dendrobium atractylodes frying Atractylodes to restore the gas of the spleen and stomach to normal for insufficient Guanmai, Adding dogwood and phellodendron cypress to the right chiro Add aconite, Cistanche cistanche, Xianling spleen, etc. to Xianhua, add keel, oyster, and ocher to restore kidney qi. Continuous treatment for 10 days is a course of treatment, with an interval of 1 day for each course, and a total of 3 courses of treatment. Observation of clinical efficacy, PSQI score and (SLC-90) score were performed on the two groups of patients before and after treatment 1 day before the start of treatment, 2 days after the end of treatment, and 1 month after stopping treatment.

\subsubsection{Observation Indicators}

Clinical efficacy, PSQI score, (SLC-90) score.

\subsubsection{Efficacy Judgment Standard}

Significantly effective: falling asleep time $<30 \mathrm{~min}$, sleep time prolonged for more than $2 \mathrm{~h}$, feel good, PSQI $\leq 7$ points; Effective: falling asleep time 30 to $45 \mathrm{~min}$, sleep time prolonged for more than $1 \mathrm{~h}$, self-feeling improved significantly, PSQI reduction rate $>30 \%$; Ineffective: Falling asleep time $>45 \mathrm{~min}$, sleep time extension $<1 \mathrm{~h}$, self-feeling has not improved, PSQI score reduction rate $<25 \%$. Total effective rate $=($ marked effect + effective $) /$ total number of cases $\times 100 \%$.

\subsubsection{Statistical Methods}

Using SPSS13.0 statistical software, measurement data were expressed as mean \pm standard deviation (), group comparisons were made by one-way analysis of variance and $t$ test, and count data were made by $\chi 2$ test.

\section{Results}

\subsection{Baseline Data}

150 Cases of Baseline Data Included in the Sample Are All Patients Who Were Hospitalized from November 2019 to June 2020 in the Prevention Treatment Center and Department of Brain Diseases, Chongqing Yongchuan Hospital of Traditional Chinese Medicine. The Demographic Data and Clinical Characteristics of the Three Groups Are Comparable $(\mathrm{P}>0.05)$, See Table 1 .

Table 1. Two groups of demographic data and clinical characteristics $(n, \bar{x})$.

\begin{tabular}{llllll}
\hline Group & $\boldsymbol{n}$ & Male/Female & Age/Year & $\begin{array}{l}\text { Average } \\
\text { Age/Year }\end{array}$ & $\begin{array}{l}\text { Course of } \\
\text { Disease (months) }\end{array}$ \\
\hline Treatment group & 50 & $18 / 32$ & $19 \sim 75$ & $57.5 \pm 18.3$ & $\begin{array}{l}\text { Average Course of } \\
\text { Disease (months) }\end{array}$ \\
Western medicine control group & 50 & $16 / 34$ & $19 \sim 75$ & $56.5 \pm 16.6$ & $0.5 \sim 20$ \\
TCM control group & 50 & $21 / 29$ & $20 \sim 71$ & $58.3 \pm 18.6$ & $0.6 \sim 23$ \\
Comparison between groups & & F- $=0.128$ & & $11.47 \pm 9.92$ & $0.4 \sim 24$ \\
P value & & $0.8803>0.05$ & & $0.8803>0.05$ & $12.53 \pm 10.54$ \\
\hline
\end{tabular}

Table 1. Continued.

\begin{tabular}{|c|c|c|c|c|c|}
\hline Group & $\begin{array}{l}\text { Deficiency of the } \\
\text { Heart and Spleen }\end{array}$ & $\begin{array}{l}\text { fire excess from } \\
\text { Yin deficiency }\end{array}$ & $\begin{array}{l}\text { timidity due to } \\
\text { deficiency of heart qi }\end{array}$ & $\begin{array}{l}\text { phlegm-fire } \\
\text { disturbing heart }\end{array}$ & $\begin{array}{l}\text { Liver-Qi } \\
\text { Stagnation }\end{array}$ \\
\hline Treatment group & 7 & 11 & 9 & 11 & 12 \\
\hline Western medicine control group & 9 & 14 & 7 & 10 & 10 \\
\hline TCM control group & 8 & 13 & 8 & 9 & 12 \\
\hline Comparison between groups & Ridit: 0.6006 & & & & \\
\hline$P$ value & $0.8963>0.05$ & & & & \\
\hline
\end{tabular}




\section{2. "Withdrawal Cases"}

During the Observation Period, 1 Case in the Treatment Group (Deficiency of the Heart and Spleen) Withdrew from Observation Due to Arrhythmia. According to the Curative Effect at the Time of Withdrawal, It Was Judged as Invalid; 50 Cases in the Control Group of Traditional Chinese and Western Medicine, There Was no Withdrawal Case During the Observation Period

\section{3. "Dropping Cases"}

During the Observation Period, There Was 1 Case of Abnormal Renal Function in the Treatment Group (Liver-Qi Stagnation), and the Treatment Was Discontinued

\subsection{Curative Effect Observation Index Analysis}

\begin{tabular}{llllll}
\hline Group & Number of cases & Remarkably effective & Effective & Ineffective & Total effective Total effective\% \\
\hline Treatment group & 48 & 26 & 16 & 6 & 87.5 \\
TCM control group & 50 & 21 & 17 & 12 & 76 \\
Western medicine control group & 50 & 19 & 18 & 13 & 74 \\
\hline
\end{tabular}

The comparison between the treatment group and the two control groups was statistically significant $(\mathrm{P}<0.05)$.

\subsection{Comparison of PSQI Scores Before and After Treatment, See Table 2 and Table 3}

Table 2. Comparison of PSQI score between insomnia treatment group and western medicine control group.

\begin{tabular}{|c|c|c|c|c|}
\hline \multirow{2}{*}{ Time point Treatment group } & \multirow{2}{*}{ Treatment group } & \multirow{2}{*}{$\begin{array}{l}\text { Western medicine control } \\
\text { group }\end{array}$} & \multicolumn{2}{|c|}{ Comparison between groups } \\
\hline & & & t & $\mathbf{P}$ \\
\hline Before treatment & $17.24 \pm 1.41$ & $16.47 \pm 1.03$ & & \\
\hline 1 month after treatment & $6.43 \pm 1.21$ & $7.43 \pm 3.45$ & 2.0507 & 0.0430 \\
\hline
\end{tabular}

The treatment group and the control group were compared between the groups after treatment, and the difference in PSGI was statistically significant $(\mathrm{P}<0.05)$.

Table 3. Comparison of PSQI score between insomnia treatment group and traditional Chinese medicine control group

\begin{tabular}{llll}
\hline Time point Treatment group & Treatment group & TCM control group & $\begin{array}{c}\text { Comparison between groups } \\
\text { P }\end{array}$ \\
\hline Before treatment & $17.24 \pm 1.41$ & $16.34 \pm 1.21$ & \\
Day 2 after treatment & $7.34 \pm 1.05$ & $8.02 \pm 1.22$ & 2.9698 \\
1 month after treatment & $6.43 \pm 1.21$ & $7.43 \pm 3.35$ & 2.0507 \\
\hline
\end{tabular}

The treatment group and the traditional Chinese medicine control group were compared between the groups after the treatment, and the difference in PSQI was statistically significant $(\mathrm{P}<0.05)$.

\subsection{Comparison of the Self-Rating Symptom Scale (SLC-90) Scores of Patients Before and After Treatment, See Table 4 and Table 5}

Table 4. Comparison of Symptom Self-rating Scale (SLC-90) before and after treatment with TCM control group.

\begin{tabular}{lllll}
\hline \multirow{2}{*}{ Time point } & Treatment group & Western medicine control group & \multicolumn{2}{c}{ Comparison between groups } \\
\cline { 4 - 5 } & & & $\mathbf{t}$ & P \\
\hline Before treatment & $201.79 \pm 42.56$ & $204.47 \pm 43.12$ & & 0.0462 \\
Day 2 after treatment & $122.53 \pm 24.54$ & $135.38 \pm 37.34$ & 2.0191 & 0.0356 \\
1 month after treatment & $115.53 \pm 27.37$ & $127.29 \pm 27.52$ & 2.1316 & \\
\hline
\end{tabular}

The self-rating symptom scale (SLC-90) scores of the treatment group and the control group were compared between the groups, and the difference was statistically significant $(\mathrm{P}<0.05)$.

Table 5. Comparison of Symptom Self-rating Scale (SLC-90) before and after treatment with the control group of TCM.

\begin{tabular}{llllc}
\hline \multirow{2}{*}{ Time point } & Treatment group & TCM control group & \multicolumn{2}{c}{ Comparison between groups } \\
\cline { 4 - 5 } & & & t & P \\
\hline Before treatment & $201.79 \pm 42.56$ & $204.47 \pm 43.12$ & 2.0122 & 0.0470 \\
Day 2 after treatment & $122.53 \pm 24.54$ & $132.32 \pm 23.87$ & 1.9999 & 0.0483 \\
1 month after treatment & $115.53 \pm 27.37$ & $126.32 \pm 26.31$ & & \\
\hline
\end{tabular}


The self-rating symptom scale (SLC-90) scores of the treatment group and the control group were compared between the groups, and the difference was statistically significant $(\mathrm{P}<0.05)$.

\section{7. "Adverse Reactions"}

There Was no Serious Adverse Reaction in Both Groups During the Observation.

\section{Conclusion}

We have compared the clinical efficacy, PSQI score and (SLC-90) score of the three groups before and after the treatment of insomnia and between the groups. Jinding Decoction had statistical significance in the treatment of insomnia of various syndrome types $(\mathrm{P}<0.05)$. Spleen and stomach harmony is the basis for the orderly rise and fall of yin and yang qi, the hidden primordial spirit, and the foundation of sleep soundness. Jinding Decoction is based on the basic theory of traditional Chinese medicine.

\section{Discussion}

A survey result released by the Chinese Sleep Research Association shows that the incidence of insomnia among adults in my country is as high as $38.2 \%$, which is higher than that in developed regions or countries such as Europe and the United States [3]. Seriously affect the daily work and quality of life of patients [4] Some studies believe that yin and yang imbalance is the total pathogenesis of insomnia. Du Yumei et al. [5] when discussing the TCM differentiation and treatment of insomnia, believe that the law of yin and yang in the human body is consistent with the yin and yang in nature The rhythm of change is consistent, and the imbalance of yin and yang in the human body can affect physiological activities such as sleep and wakefulness. Huang Yuanyu proposed: The person with Qi of the Middle Energizer is the pivot of yin and yang, the so-called The Speen Earth. Water, Fire, Gold and Wood are the four elephants. The four elephants are the rise and fall of yin and yang, and yin and yang are the rise and fall of qi. According to the traditional Chinese medicine theory "harmonizing stomach to tran-quilize the mind and the theory that disharmony of stomach leads to insomnia", and the western medicine brain-intestinal theory: the gastrointestinal nervous system is the human "second brain", the traditional Chinese medicine theory of the spleen and stomach center and Western medicine is closely related to the gastrointestinal nerves. Based on the traditional spleen and stomach central theory of traditional Chinese medicine, the gastrointestinal nervous system is the human "second brain" as the guiding ideology, and further explores Clinical Study on different types of insomnia based on Conducting yin from yang, conducting yang from yin Method with Spleen and Stomach as Center.

Traditional Chinese medicine has a history of treating insomnia for more than two thousand years. According to Chinese medicine, insomnia belongs to the range of "palpitations," "insomnia," and "psychosis" [6]. The pathogenesis of insomnia in Chinese medicine is mainly: Ying-Wei Yin-Yang Theory, Zang-Fu Theory, Phlegm and Blood Stasis Theory, and Emotion Theory. The Yin-Yang theory of Ying-Wei believes that the imbalance of Ying-Wei and Yin-Yang can cause insomnia, imbalance of Yin-Yang [7], or yin deficiency cannot absorb yang, or yang cannot enter yin. In the opening and closing of yin and yang, the transfer of spleen soil plays an important role. The viscera theory believes that insomnia is closely related to the heart, liver, spleen and stomach, gallbladder, brain, etc. The spleen soil, as the hub of the various viscera, plays an important pivotal role of "the stomach is not in harmony with the restlessness". The spleen and stomach are the "foundation of the acquired nature", and the stomach is the "sea of the five internal organs". The spleen and stomach infuse water and grains into the heart, lungs, and liver to nourish the whole body. "Stomach qi" is the foundation of human beings, "if there is stomach qi, it is born" [8].

"People receive qi from the food, and the food enters the stomach, to pass it to the lungs, the internal organs, and the internal organs. All are based on receiving qi. The lighter is the camp, and the turbid one is the guard. In the reunion meeting, the yin and yang are intertwined, like a ring for no reason." [9] It can be seen that "protecting stomach qi" is an important principle in TCM treatment. The "harmony" and "disharmony" of the stomach are particularly closely related to "sleeping restlessness". The treatment of insomnia symptoms by regulating the stomach reflects the idea of "seeking the root of disease" in traditional Chinese medicine [10]. "The Second Classic" says: harmonizing stomach to tranquilize the mind and the theory that disharmony of stomach leads to insomnia. "[11] Wang Hongtu [12] put forward that the spleen and stomach are in the center, which is the pivot of the vital energy movement, and the normal operation of the guarding qi! If it does not enter the Yin meridian, insomnia will occur. "God, the essence of water valley", so many classic insomnia theories, such as "yin and yang theory", "shenzhu theory", "qi and blood theory", "yingwei theory" are closely related to the "stomach" of traditional Chinese medicine [13]. For example, the loss of yin essence, poor sealing power can not communicate with the heart and kidney [14], liver and gallbladder dysfunction, abnormal spleen mobilization, poor blood production affect sleep, and other changes in the function of viscera will affect sleep. The problem of insomnia is that the yin and yang rise and fall. The spleen and stomach play an important pivotal role in the process of rising and exiting. The gastrointestinal tract is the human's second brain [15] (abdominal brain or intestinal brain), which is the intestine The nervous system is separated from the independent activity system innervated by the central nervous system. In 1965, Gershon published an article in the "Science" magazine and proposed that 5-HT may act as a neurotransmitter in the intestine. Almost all transmitters and modulators in the central nervous system are present in the enteric nervous system, and the brain [16] regulates enteric nervous system activities through the input or output of sympathetic or parasympathetic nerves. The gastrointestinal nervous system is the "second brain" of humans. The traditional 
spleen and stomach central theory of Chinese medicine is closely related to the western medicine gastrointestinal nerves [17]. Based on the traditional Chinese medicine spleen and stomach central theory, the gastrointestinal nervous system is the human "second brain" For the guiding ideology, further explore the research on different syndrome types of insomnia by using the spleen and stomach as the center of Yin and Yang. Take "Four Sacred Heart Source" Jinding Decoction (Glycyrrhiza 6g Poria 9g Pinellia 3g Cinnamon sticks 9g Peony 9g keel 6g Oyster 9g addition and subtraction, Pinellia reduces stomach soil, Guizhi reaches the stagnation of the liver, Paeonia suffocating gallbladder fire, dragon bone, oyster storage Focusing on the yang roots. When yang descends deep from the roots, the soul is calm and tranquil. Separate the syndromes without isolation, and establish a true central framework of the spleen and stomach.

\section{Competing Interests}

All the authors do not have any possible conflicts of interest.

\section{Authors' Contributions}

Bian wei: data collection, background research, Wang huanqun: treatment recording writing; Gan ting: $\mathrm{R}$ data analysis; Zou weiwu: therapeutic assessment;

Bian wei: performed treatments, overall responsibility. All the authors read and approved the final report.

\section{Acknowledgements}

The authors are grateful for the support of Chongqing Yongchuan Hospital of Traditional Chinese Medicine.

The research of this subject is funded by the special fundof Chongqing Science and Technology Bureau of Yongchuan.

District. The approval number of this subject is: Ycstc, 2019 nb0230.

\section{References}

[1] Du Yumei. Discussion on TCM syndrome differentiation and treatment of insomnia (Chinese medicine insomnia) [J]. Psychologist, 2017, 23 (17): 133-134.

[2] Psychiatric Branch of Chinese Medical Association. Classification and Diagnostic Criteria of Mental Diseases in China [M]. Third Edition. Shandong: Shandong Science and Technology Press, 2001: 118.

[3] State Administration of Traditional Chinese Medicine. Standards for Diagnosis and Efficacy of TCM Diseases and Syndromes [S]. Nanjing: Nanjing University Press, 2012: 19.
[4] Research Group of Clinical Practice Guidelines for Insomnia, College of Chinese Medicine and Chinese Medicine. Clinical Practice Guidelines of Traditional Chinese Medicine for Insomnia (WHO/WPO) [J]. World Journal of Sleep Medicine, 2016, 3 (1): 8-25.

[5] Lai Aiqun, He Yuqiu, Luo Yuling, et al. Investigation of mental health status of patients with insomnia [J]. Journal of Clinical Psychosomatic Diseases, 2015, 22 (3): 71-73.

[6] Yang Fang, Sun Lixia, Qin Xia. Observation on the efficacy of traditional Chinese medicine in treating patients with insomnia in neurology $[\mathrm{J}]$. Clinical Research of Traditional Chinese Medicine, 2016. 8 (24): 95-96.

[7] Chen Yi: Research progress in the treatment of insomnia with traditional Chinese medicine [J]. Clinical Research of Chinese Medicine, 2017. 9 (7): 138-140.

[8] Lu Junzhang, Yang Ming. The theory of gastric qi and its clinical application principles $[\mathrm{J}]$. Chinese Journal of Traditional Chinese Medicine, 2005, 20 (4): 201-203.

[9] Zhai Shuangqing. Selected Readings of Nei Jing [M]. Beijing: China Press of Traditional Chinese Medicine, 2013: 124-125.

[10] Zhao Wenhui, Jiang Shumin. Analysis and clinical experience of "Stomach discord causes restlessness" [J]. Clinical Journal of Traditional Chinese Medicine. 2006, 28 (1): 24-26.

[11] Tang Wang Bing. Huangdi Neijing.Suwen [M]. Beijing: People's Medical Publishing House, 2002: 435.

[12] Wang Hongtu, He Juan, Zhai Shuangqing, et al. A review of the research on the regulation of the five zang organs by the spleen and stomach circulatory system $[\mathrm{J}]$. Journal of Beijing University of Traditional Chinese Medicine, 2002, 25 (2): 1-4.

[13] Li Shaodan, Yang Minghui. Clinical study on the treatment of sub-healthy insomnia by Hewei Anshen [J]. Journal of Clinical Rational Use, 2011, 4 (27): 69-71.

[14] Hu Zhanyun, Zhang Shizang. Analysis of clinical efficacy of traditional Chinese medicine in the treatment of neurological insomnia patients $[\mathrm{J}]$ Clinical Research of Traditional Chinese Medicine. 2015. 7 (6): 13-14.

[15] Tian Zaishan, Wu Xianzhong, Chen Yufeng. About "abdominal brain (second brain)" [J]. Chinese Journal of Integrated Traditional Chinese and Western Medicine Surgery, 2005, 11 (5): 454-457.

[16] Zhang Yanmei, "Thinking about hurting the spleen" and "Brain-gut peptide" [J]. Chinese Journal of Basic Medicine in Traditional Chinese Medicine, 2000, 6 (1): 6-7.

[17] Wang Huanqun, Bian Wei. Case study of treating insomnia with spleen and stomach twisting combined with five internal organs [J]. World Journal of Sleep Medicine, 2019, 6 (1): 43-44. 